\title{
Effects of branched or linear architecture of bioreducible poly(amido amine)s on their in vitro gene delivery properties
}

\author{
Federico Martello ${ }^{a}$, Martin Piest ${ }^{b}$, Johan F.J. Engbersen ${ }^{\text {b,* }}{ }^{\text {, Paolo Ferruti }}{ }^{\text {a }}$ \\ a Dipartimento di Chimica Organica e Industriale, Universitá degli Studi di Milano, via Venezian 21, 20133 Milan, Italy

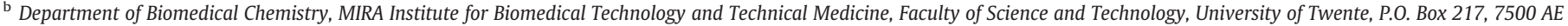 \\ Enschede, The Netherlands
}

\section{A R T I C L E I N F O}

\section{Article history:}

Received 27 May 2012

Accepted 21 July 2012

Available online $\mathrm{xxxx}$

\section{Keywords:}

Michael addition

Hyperbranched poly(amido amine)

Gene delivery

Polyplex

Disulfide reduction

\begin{abstract}
A B S T R A C T
In this study, the gene delivery properties of new hyperbranched poly(amido amine)s (PAAs) with disulfide linkages in the main chain were investigated in comparison with their linear analogs. Eight different bioreducible PAAs were prepared by Michael addition of $N, N^{\prime}$-bisacryloylpiperazine (BP) with cystamine (CYST) or $N, N^{\prime}$-dimethylcystamine (DMC) and of $N, N^{\prime}$-cystaminebisacrylamide (CBA) with $N, N^{\prime}$-ethylenediamine (EDA) or $N, N^{\prime}$-dimethylethylenediamine (DMEDA). In order to study the effect of terminal groups on the transfection efficiency, each polymer was terminated with 4-aminobutanol (ABOL) or with 2-aminoethanol (ETA). The hyperbranched and the linear PAAs generally formed polyplexes with plasmid DNA with sizes around $200 \mathrm{~nm}$ and positive zeta potentials ranging from +10 to $+22 \mathrm{mV}$ at polymer/DNA weight ratios equal or higher than 3/1. Remarkably low or no cytotoxicity was observed for both hyperbranched and linear PAAs. Hyperbranched CBA-containing PAAs showed higher gene expression in DNA transfection tests with COS-7 cells than their linear analogs and up to two times higher than linear PEI that was used as the reference polymer. Transfection efficiencies of the branched PAAs were generally enhanced by the presence of serum, which is a promising property for future in vivo studies with these hyperbranched PAAs.

In this study the ease of synthetic modification of both linear and hyperbranched poly(amido amide)s and the versatility of hyperbranched PAAs in regulating DNA transfection and cytotoxicity are demonstrated. The results show the large possibilities for this class of polymers to provide polymeric vectors with controllable properties for gene therapy applications.
\end{abstract}

(c) 2012 Elsevier B.V. All rights reserved.

\section{Introduction}

The main challenge in gene therapy is successful in vivo transfer of genetic materials to the targeted tissues. Unfortunately, a suitable vector for safe and efficient delivery of therapeutic genes into target cells is currently not yet available [1-4]. Vectors for gene delivery can be divided into two classes, viral and non viral. Viral vectors are obtained by modification of viruses to allow the delivery of foreign DNA. Although viral vectors possess high transfection efficiencies, they have some major drawbacks, like low gene loading capacity, limitations to repeated administration due to immune response and difficulties in production and quality control [5-9]. In contrast, non-viral vectors such as lipoplexes and cationic polymer DNA complexes (polyplexes) have lower host immunogenicity, enabling repeated administration, higher gene carrying capacity, and can be obtained with simple large scale synthesis, although they possess generally a lower efficiency in vivo as compared to viral vectors [10-17].

\footnotetext{
* Corresponding author. Tel.: + 3153 4892926; fax: + 31534892155 .

E-mail address: j.f.j.engbersen@tnw.utwente.nl (J.F.J. Engbersen).
}

In particular, poly(amido amine)s (PAAs) have shown to be as a versatile class of promising polymers for biomedical application, owing to different qualities including biodegradability, biocompatibility, generally low hemolytic activity, good water solubility and peptide-mimicking structures [18]. For example, in gene delivery, several amphoteric PAAs carrying a carboxylic acid side group in the bisacrylamide segment showed good endosomolytic properties [19] as well as transfection efficiencies comparable to polyethylenimine (PEI) [20]. Their good endosomolytic properties can be explained by the capacity of these polymers to undergo conformational changes in the endosomal $\mathrm{pH}$ range. Furthermore, the introduction of bioreducible disulfide linkages in the main chain facilitates a rapid vector unpacking and DNA release in a reductive environment, such as the cytosol where glutathione levels are thousand-fold higher than in the extracellular environment. Moreover, disulfide reduction allows for rapid polymer degradation into small non-toxic fragments. The incorporation of bioreducible disulfide linkages in polymers for gene and drug delivery has been favorably exploited by us and several other groups [21-24].

In our group, a library of PAAs containing disulfide linkages in the main chain (SS-PAAs) carrying various side chains including oligoamine side chains, or DNA intercalating side chains has been developed and 
the structure-function relationships of SS-PAAs for optimal DNA transfection have been thoroughly investigated [25-30]. Most importantly, it was found that the bioreducible PAAs containing disulfide linkages in the main chain have a DNA transfection efficiency that is generally higher than PEI, being considered the gold standard in polymeric gene transfection. These polymers were also generally more efficient in DNA transfection than their analogs lacking disulfide linkages [25]. This can be ascribed to the biodegradability of the disulfide group, and therewith of the polymer, in the intracellular environment. It was also found that, despite their cationic nature, the SS-PAAs have little or no cytotoxicity. In a recent study of Oupicky and coworkers, it was shown that cytotoxicity of hyperbranched bioreducible PAAs is directly related to their ability to degrade intracellularly and decreases with increasing the disulfide content [31].

With respect to branched architectures, it has been demonstrated that partially degraded poly(amido amine) dendrimers ("activated PAMAM") were more efficient in DNA transfection than nondegraded dendrimers [32]. This is likely due to the higher structural flexibility in solution of partially degraded dendrimers. In a more recent study, it was found that hyperbranched poly(ester amine)s transfect DNA into COS-7 cells with higher efficiency than PEI and poly(2-(dimethylamino)ethyl methacrylate) (pDMAEMA) [33]. Moreover, these hyperbranched poly(ester amine)s showed little or no toxicity in vitro. In a recent work of Wang and co-workers it has been observed that by increasing the branching degree of PAAs the transfection efficiency increases and the cytotoxicity decreases. In this case a transfection efficiency comparable to PEI was obtained using the PAA with the highest degree of branching [34]

Therefore, we hypothesized that hyperbranched PAAs containing disulfide linkages in the main chain could have advantages in DNA transfection compared to traditional linear PAAs. Firstly, the high density of secondary and tertiary amines in the polymer increases the buffering capacity over a wide $\mathrm{pH}$ range, which may result in promotion of the endosomal escape of the polymer/DNA complexes by the proton sponge effect [35] and increased disruptive interactions with the endosomal membrane. Secondly, due to their easy synthesis, hyperbranched PAAs could be used as platform to systematically investigate the effect of terminal groups. In hyperbranched polymers the ratio between the number of terminal functions and the total number of monomeric units is significantly higher than in linear polymers. Thus, by selecting suitable terminal groups for the hyperbranched PAA polymers it is possible to improve the solubility in water, adjust the interaction between polymer and DNA in the polyplexes, and the interaction of the polyplexes with their biological environment in the transfection process. In this respect, it was found by Langer and co-workers that the terminal groups of linear poly(amino esters) can significantly alter the transfection efficiency, although the exact relationship between the structure of terminal unit and the transfection efficiency is not clear [36]. Thirdly, by introducing disulfide linkages in the hyperbranched polymer chains, rapid degradation into small non-toxic fragments is achieved in the reductive intracellular environment, resulting in good unpacking of the therapeutic payload from these polymeric carriers and low cytotoxicity effects.

Based on these premises, we have synthesized a new series of biodegradable SS-PAAs with a linear or a branched architecture (Scheme 1) and tested their biological properties as well as the DNA transfection efficiency of polymer-DNA polyplexes.

\section{Experimental part}

\subsection{Materials}

All solvents were of analytical grade purchased from Aldrich or Fluka and used as received. Methanol $(\mathrm{MeOH})$, sodium hydroxide $(\mathrm{NaOH}), 37 \%$ hydrochloric acid ( $\mathrm{HCl} 37 \%), 1,2$-diaminoethane (EDA), triethylamine (TEA), cesium bicarbonate $\left(\mathrm{Cs}_{2} \mathrm{CO}_{3}\right), N, N^{\prime}$-dimethylethylenediamine (DMEDA), N,N'-bisacryloylcystamine (CBA), cystamine dihydrochloride

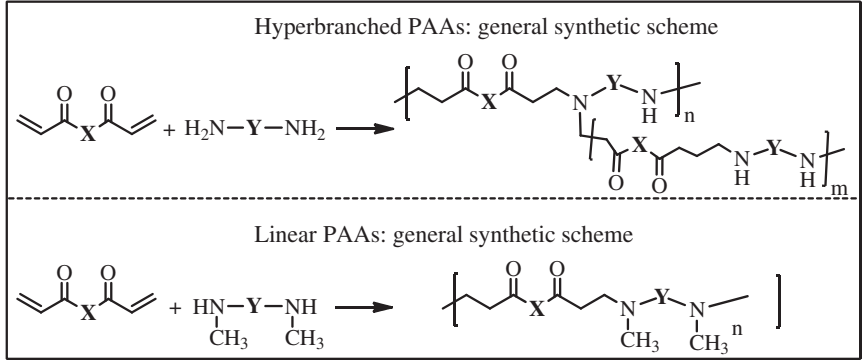

\begin{tabular}{|c|c|c|}
\hline Bisacrylamide & Amine & $+\begin{array}{c}\text { End-capping } \\
\text { amine }\end{array}$ \\
\hline & $\mathrm{H}_{2}^{\mathrm{H}_{2} \mathrm{~N} \sim \mathrm{S}_{\mathrm{SST}}}$ & 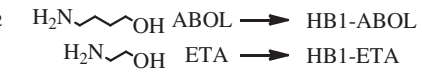 \\
\hline BP & $\underset{\mathrm{H}}{\mathrm{N}} \mathrm{NMC}_{\mathrm{S}}^{\mathrm{S}_{\mathrm{S}}}$ & 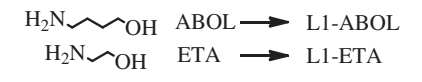 \\
\hline & $\underset{\text { EDA }}{\mathrm{H}_{2} \mathrm{~N} \sim \mathrm{NH}_{2}}$ & 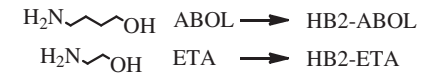 \\
\hline $\mathrm{H}$ CBA & ${\underset{\mathrm{DMEDA}}{\mathrm{H}}}_{\mathrm{N}}^{\sim \mathrm{H}}$ & $\begin{array}{l}\mathrm{H}_{2} \mathrm{~N} \sim \mathrm{OH} \mathrm{ABOL} \longrightarrow \text { L2-ABOL } \\
\mathrm{H}_{2} \mathrm{~N} \sim \mathrm{OH} \text { ETA } \longrightarrow \text { L2-ETA }\end{array}$ \\
\hline
\end{tabular}

Scheme 1. General synthetic schemes and monomers utilized to obtain hyperbranched and linear PAAs. Abbreviations: HB, hyperbranched; L, linear; BP, $N, N$ '-bisacryloylpiperazine; CBA, N,N'-bisacryloylcystamine; CYST, cystamine; DMC, $N, N$ '-dimethylcystamine; EDA, ethylenediamine; DMEDA, $N, N^{\prime}$-dimethylethylenediamine; ABOL, 4-aminobutanol; ETA, 2-aminoethanol.

(CYST), 4-aminobutanol (ABOL), 2-aminoethanol (ETA) were purchased from Fluka at the highest purity and used without further purifications. Exgen 500 was purchased from Aldrich and used without further purifications. The plasmid pCMV-LacZ, containing a bacterial LacZ gene preceded by a nuclear localization signal under control of a CMV promoter, was purchased from Plasmid Factory (Bielefeld, Germany).

\subsection{Methods}

${ }^{1} \mathrm{H}$ NMR spectra were recorded on Bruker spectrometers operating at $400 \mathrm{MHz}$. Polymer samples were prepared by dissolving polymer (in salt-free form, about $10 \mathrm{mg}$ ) in $1 \mathrm{~mL}$ of $\mathrm{D}_{2} \mathrm{O} .{ }^{1} \mathrm{H}$ NMR spectra are given in the Supplementary Materials section. Polymer molecular weight $\left(\mathrm{M}_{\mathrm{w}} / \mathrm{M}_{\mathrm{n}}\right)$ and polydispersity (PD) of the synthesized poly(amido amine)s were determined by Gel Permeation Chromatography (GPC) using a Knauer Pump 1000 equipped with a Knauer Autosampler 3800, TSKgel G4000 PW and G3000 PW TosoHaas columns connected in series, Light Scattering (LALS) Viscotek 270 Dual Detector, a UV detector Waters model 486, operating at $230 \mathrm{~nm}$ and a Refractive Index detector Waters model 2410. The mobile phase was a 0.1 Tris buffer $\mathrm{pH} 8.00 \pm 0.05$ with $0.2 \mathrm{M}$ sodium chloride.

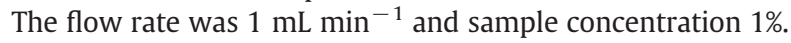

\subsubsection{Reductive degradation studies of PAAs}

The selected PAA (700 mg, $0.4 \mathrm{mmol}$ of repeating unit) was dissolved in a $\mathrm{D}_{2} \mathrm{O}(4 \mathrm{~mL})$. The homogeneous solution obtained was maintained at $25{ }^{\circ} \mathrm{C}$ under nitrogen atmosphere while stirring, and then 2-mercaptoethanol ( $70 \mathrm{mg} ; 0.88 \mathrm{mmol}$ ) added. An aliquot of solution (1 mL) was taken after 30 min and analyzed by ${ }^{1} \mathrm{H}$ NMR [37].

\subsubsection{Buffer capacity}

The buffer capacity of the PAA polymers was determined by acidbase titration. An amount equal to $5 \mathrm{mmol}$ of amine groups of the PAA polymer was dissolved in $10 \mathrm{~mL}$ of $150 \mathrm{mM} \mathrm{NaCl}$ aqueous solution. The $\mathrm{pH}$ of the polymer solution was set at 2.0 and the solution was titrated with $0.1 \mathrm{M} \mathrm{NaOH}$ solution using an automatic titrator (Metrohm 
702 SM Titrino). For comparison, linear PEI ( $\left.\mathrm{M}_{\mathrm{w}}=22 \mathrm{kDa}\right)$ dissolved in $150 \mathrm{mM}$ aqueous solution adjusted to $\mathrm{pH} 2.0$, was also titrated using the same method. The buffering capacity defined as the percentage of amine groups becoming protonated from $\mathrm{pH} 5.1$ to 7.4, was calculated from Eq. (1):

Buffer capacity $(\%)=\left(\Delta V_{\mathrm{NaOH}} \times 0.1\right) / N$

Wherein $\Delta V_{\mathrm{NaOH}}$, is the volume of $\mathrm{NaOH}$ solution $(0.1 \mathrm{M})$ required to bring the $\mathrm{pH}$ value of the polymer solution from 5.1 to 7.4, and $N$ is the total moles ( $5 \mathrm{mmol}$ ) of protonable amine groups in PAA polymer.

\subsubsection{Particle size and zeta-potential measurements}

The surface charge and the size of polyplexes were measured at $25{ }^{\circ} \mathrm{C}$ with a Dynamic Light Scattering (DLS) system Zetasizer 4000 (Malvern Instruments Ltd., Malvern, UK). PAA/plasmid DNA polyplexes at different polymer/DNA weight ratios were prepared by adding a HEPES buffer solution (20 mM, pH 7.4, 5 wt.\% glucose) of poly(amido amine)s ( $800 \mu \mathrm{L})$ to a HEPES buffer solution of plasmid DNA ( $200 \mu \mathrm{L}$, $75 \mu \mathrm{g} / \mathrm{mL}$ ), followed by vortexing for $5 \mathrm{~s}$ and incubating at room temperature for $30 \mathrm{~min}$.

\subsubsection{Agarose gel retardation assay}

Polyplexes were prepared at 3/1,6/1, 12/1, and 48/1 polymer/DNA weight ratio respectively in HEPES ( $20 \mathrm{mM}$, pH 7.4). Next, ethidium bromide $\left(2.5 * 10^{-6} \mathrm{M}\right.$ final concentration) was added to the polyplexes and the polyplexes were incubated for another $30 \mathrm{~min}$ at ambient temperature. Plasmid DNA (positive control, $0.015 \mathrm{mg} / \mathrm{mL}$ ) and the polyplexes ( $25 \mu \mathrm{L}$ ) were mixed with $5 \mu \mathrm{L}$ of loading buffer and $25 \mu \mathrm{L}$ of each sample was loaded on a $0.8 \% \mathrm{w} / \mathrm{v}$ agarose gel containing $0.5 \mu \mathrm{g} / \mathrm{mL}$ ethidium bromide $(1.25 \mu \mathrm{M})$. Electrophoresis was performed for $60 \mathrm{~min}$ at $90 \mathrm{~V}$ in a TAE running buffer ( $40 \mathrm{mM})$ tris(hydroxymethyl)aminomethane, $20 \mathrm{mM}$ acetic acid, $10 \mathrm{mM}$ EDTA, pH 8,0) supplemented with $1.25 \mu \mathrm{M}$ ethidium bromide. After electrophoresis pictures were taken with a BioRad Gel Doc 2000 under UV-illumination, using Multi-Analyst software v.1.1.

\subsubsection{In vitro transfection and cell viability assays}

Transfection experiments were performed with COS-7 cells (SV-40 transformed African Green monkey kidney cells) by using the plasmid pCMV-LacZ [38,39]. Two parallel transfection series, one for the determination of reporter gene expression ( $\beta$-galactosidase) and the other for the evaluation of cell viability by XTT assay, were carried out in separate 96-well plates (ca. $1.0 \times 10^{4}$ cells per well). Different polymer/plasmid DNA weight ratios, ranging from $3 / 1$ to $48 / 1$, were used to prepare the polyplexes. In brief, polyplexes were prepared by adding $200 \mu \mathrm{L}$ of a HEPES buffer solution ( $20 \mathrm{mM}, 130 \mathrm{mM} \mathrm{NaCl}, \mathrm{pH} 7.4$ ) of poly(amido amine) with varying concentrations (from 9.4 to $150 \mu \mathrm{g} / \mathrm{mL}$ ) to $50 \mu \mathrm{L}$ of a HEPES buffer solution of plasmid DNA $(50 \mu \mathrm{g} / \mathrm{mL})$, followed by gentle shaking and incubating at room temperature for $30 \mathrm{~min}$. All transfection and toxicity assays were carried out with $n=3$. In a standard transfection experiment, the cells were incubated with the desired amount of polyplexes ( $100 \mu \mathrm{L}$ dispersion with $1 \mu \mathrm{g}$ plasmid DNA per well) for $1 \mathrm{~h}$ at $37{ }^{\circ} \mathrm{C}$ in a humidified $5 \% \mathrm{CO}_{2}$-containing atmosphere. Next, the polyplexes were removed. $100 \mu \mathrm{L}$ of fresh culture medium was added and the cells were cultured for 2 days. The transfection efficiency was determined by measuring the activity of $\beta$-galactosidase using the ONPG assay [38]. Exgen500 was used as a reference (linear PEI formulation prepared at its optimal $\mathrm{N} / \mathrm{P}$ ratio of 5 , according to manufacturer). The number of viable cells was measured using an XTT assay [40]. The XTT value for untreated cells (i.e. cells not exposed to the transfection medium) was taken as $100 \%$ cell viability.

\subsubsection{Synthesis of the monomers}

$N, N^{\prime}$-dimethylcystamine (DMC) and 1,4-bisacryloylpiperazine (BP) were synthesized as previously described (BP, [41] DMC, [27]), and their purity (>99\%) was determined by ${ }^{1} \mathrm{H}$ NMR spectroscopy prior to use.

\subsubsection{Synthesis of HB1-based polymers}

CYST $(0.31 \mathrm{~g} ; 1.35 \mathrm{mmol})$ and BP ( $1.69 \mathrm{~g} ; 8.72 \mathrm{mmol})$ were dissolved in water $(5.0 \mathrm{~mL})$ in a $10 \mathrm{~mL}$ round-bottom flask. The $\mathrm{pH}$ was then adjusted to 9.0-9.5 by adding $\mathrm{Cs}_{2} \mathrm{CO}_{3}$. The reactant solution was stirred at room temperature for 3 days under inert atmosphere and in the dark. The solution was then diluted with $20 \mathrm{~mL}$ of water, acidified to $\mathrm{pH} 4.5$ with a few drops of $\mathrm{HCl}(37 \%)$, ultrafiltrated (1000 Da cut-off membrane) and freeze-dried. Afterwards, the polymer $(0.51 \mathrm{~g} ; 1.48 \mathrm{mmol}$ of repeating units) was dissolved in $\mathrm{MeOH}(5.20 \mathrm{~mL}$ ) and then $40 \mathrm{~mol} \%$ excess of $\mathrm{ABOL}$ (to obtain HB1-ABOL) or ETA (to obtain HB1-ETA) was added to consume the terminal acrylamide groups and stirring was continued for $24 \mathrm{~h}$ at ambient temperature. Finally, the solution was diluted with water $(20 \mathrm{~mL})$, acidified to $\mathrm{pH} 4.5$ with $\mathrm{HCl}(37 \%)$, ultrafiltrated (1000 Da cut-off membrane) and freeze-dried. Yield 40\% w/w. Insoluble aggregates were eliminated by ultrafiltration (100,000 Da cut-off membrane).

Synthesis of L1-based polymers: in a $10 \mathrm{~mL}$ round-bottom flask, double distilled water $(1.80 \mathrm{~mL}), N, N^{\prime}$-dimethylcystamine dihydrocloride (DMC) (0.60 g; $2.37 \mathrm{mmol})$ and BP (0.46 g; $2.37 \mathrm{mmol})$ were added sequentially. The $\mathrm{pH}$ was then adjusted to ca. 10 by adding TEA $(0.48 \mathrm{~g} ; 4.76 \mathrm{mmol})$. The reactant solution was stirred at room temperature for 3 days under inert atmosphere and in the dark. Afterwards, $10 \mathrm{~mol} \%$ excess of $\mathrm{ABOL}$ (to obtain L1-ABOL) or ETA (to obtain L1-ETA) was added to consume any unreacted acrylamide groups and stirring was continued for $24 \mathrm{~h}$. The solution was then diluted with $20 \mathrm{~mL}$ of water, acidified at $\mathrm{pH} 4.5$ with $\mathrm{HCl}$ (37\%), ultrafiltrated (1000 Da cut-off membrane) and freeze-dried. Yield $87 \% \mathrm{w} / \mathrm{w}$.

Synthesis of HB2-based polymers: in a $10 \mathrm{~mL}$ round-bottom flask, CBA (2, $14 \mathrm{~g} ; 8.23 \mathrm{mmol}$ ), a solution $\mathrm{MeOH} /$ bidistilled water 5.395/1 and EDA (purity 99.5\%; 0,082 g; $1.36 \mathrm{mmol}$ ) were added sequentially under stirring. The $\mathrm{pH}$ was then adjusted to ca. 10 by adding TEA ( $0.48 \mathrm{~g} ; 4.76 \mathrm{mmol}$ ). The reaction was carried out at $40{ }^{\circ} \mathrm{C}$ for 5 days. The solution was then diluted with $20 \mathrm{~mL}$ of water, acidified to $\mathrm{pH} 4.5$ with a few drops of $\mathrm{HCl}$ (37\%), ultrafiltrated (1000 Da cut-off membrane) and freeze-dried. Afterwards, the polymer ( $0.50 \mathrm{~g} ; 1.56 \mathrm{mmol}$ of repeating units) was dissolved in $\mathrm{MeOH}(5.20 \mathrm{~mL})$ and then $40 \mathrm{~mol} \%$ excess of ABOL (to obtain HB2-ABOL) or ETA (to obtain HB2-ETA) was added to consume the terminal acrylamide groups and stirring was continued for $24 \mathrm{~h}$ at ambient temperature. Finally, the solution was diluted with water $(20 \mathrm{~mL})$, acidified to $\mathrm{pH} 4.5$ with $\mathrm{HCl}$ (37\%), ultrafiltrated (1000 Da cut-off membrane) and freeze-dried. Yield $38 \% \mathrm{w} / \mathrm{w}$. Insoluble aggregates were eliminated by ultrafiltration (100,000 Da cut-off).

Synthesis of L2-based polymers: in a $10 \mathrm{~mL}$ round-bottom flask, CBA (1.49 mg; $5.72 \mathrm{mmol}$ ), a solution $\mathrm{MeOH} /$ bidistilled water 4.636/1 (4.34 mL) and DMEDA (purity: 99\%; $0.51 \mathrm{mg} ; 5.72 \mathrm{mmol}$ ) were added sequentially under stirring. The $\mathrm{pH}$ was then adjusted to ca. 10 by adding TEA ( $0.48 \mathrm{~g} ; 4.76 \mathrm{mmol})$. The reaction was carried out at $40{ }^{\circ} \mathrm{C}$ for 5 days. Afterwards, $10 \mathrm{~mol} \%$ excess of $\mathrm{ABOL}$ (to obtain L2-ABOL) or ETA (to obtain L2-ETA) was added to consume any unreacted acrylamide groups and stirring was continued for $24 \mathrm{~h}$. The solution was then diluted with $20 \mathrm{~mL}$ of water, acidified at $\mathrm{pH} 4.5$ with $\mathrm{HCl}$ (37\%), ultrafiltrated (1000 Da cut-off membrane) and freeze-dried. Yield 75\% w/w.

\section{Results and discussion}

\subsection{PAAs synthesis and characterization}

In our previous work we have developed different series of poly(amido amine)s with disulfide linkages in the main chain (SS-PAAs) 
that showed very promising properties in DNA transfection [25-30]. In these studies, the polymer-DNA polyplexes formed with SS-PAAs containing hydroxybutyl side chains showed transfection efficiencies more than three times higher than polyethyleneimine (PEI) and lower cytotoxicity than PEI. Although the exact relationship between the side group and the transfection efficiency is still not clear, the length of the alkyl chain and the nature of the substituent at the side chains appeared to be significant for achieving a desirable hydrophobic/hydrophilic balance in the polymer.

The aim of this study was to investigate the effects of hyperbranched architecture and end-capping of these structures on the DNA transfection properties compared to their linear analogs. Based on the structures of the most efficient SS-PAAs for DNA delivery that were previously developed, we have prepared a small library of branched and linear PAA polymers containing disulfide linkages in either their bisacrylamide or amine fragments by Michael-type polyaddition of the appropriate bisacrylamides and amines. Reaction of secondary diamines with bisacrylamides leads to linear polymers since a secondary amine group can only react once as a Michael donor, whereas the primary amine groups of a primary diamine can each participate twice, leading to branched structures. To obtain the branched and linear PAA polymers with the disulfide groups in the amine fragments, $N, N^{\prime}$-bisacryloylpiperazine (BP) was reacted with the disulfide-containing amines cystamine (CYST) and $N, N^{\prime}$-dimethylcystamine (DMC), respectively. Similarly, for the branched and linear PAA polymers with the disulfide groups in the bisacrylamide fragments $N, N^{\prime}$-bisacryloylcystamine (CBA) was reacted with ethylenediamine and $N, N^{\prime}$-ethylenediamine (EDA), respectively. All these polymers were end-capped with either 4-aminobutanol (ABOL) or with 2-aminoethanol (ETA) in the final step of their synthesis, yielding eight different biodegradable PAAs (Scheme 1). All synthesized polymers were characterized by gel permeation chromatography (GPC) and ${ }^{1} \mathrm{H}$ NMR spectroscopy.

The linear PAAs were synthesized according to a literature procedure [18]. In the case of HB2 the polymerization reaction was carried out in a $4 / 1$ methanol/water mixture at $40{ }^{\circ} \mathrm{C}$, since $\mathrm{CBA}$ monomer is poorly soluble in water. As secondary diamines, DMC and DMEDA were chosen because of their structural similarity with the primary diamines CYST and EDA used for the syntheses of the hyperbranched polymers.

In the polymerization of CYST and EDA, which react as tetrafunctional monomers, both primary and secondary amine groups are involved, whereas in the polymerization of DMC and DMEDA reaction proceeds by addition of secondary amine groups only. If in the former case the primary and secondary amines of the partially reacted tetrafunctional monomers have equal reactivity, infinite networks as manifested by gelation are formed with predictable gel points $[42,43]$. In our case, however, the reactivity of tetrafunctional groups is unequal and many possible reaction routes exist. In particular, the addition rate of the primary amino groups to the double bond is higher than that of the secondary amino group generated from the first addition. The lower reaction rate related to the third and the fourth additions is presumably biased by a combination of steric hindrance and decrease in nucleophilicity due to substituents carrying electron-withdrawing carbonyl groups in the $\beta$ position. Therefore, the polymerization reaction can be divided in three phases; in the first phase a linear chain growth is prevailing, in the second phase branching occurs, and this can even lead to crosslinking in the third phase. The second and third phases will occur only at high conversion.

In the recent work of Ferruti, Ranucci, Trotta and Cavalli [44] a new approach was studied to determine the reactivity of different functionalities within multifunctional monomers in order to obtain hyperbranched PAAs. In particular, stoichiometrically imbalanced reactant mixtures with excess of double bonds were used to adjust the degree of branching of the polymers. To determine the critical stoichiometric ratio " $r_{c}$ ", under which the system is unable to gel and yields hyperbranched but still soluble polymers, Eq. (2) was used [42]:

$r_{c}=\frac{1}{1+\rho(f-2)}$

Where:

$r_{c} \quad$ critical stoichiometric ratio (number of functions in defect/ number of functions in excess).

$\rho \quad$ the fraction of functions belonging to the monomer with functionality $>2$ with respect to the total amount of the functions of the same type initially present in the system.

$f \quad$ number of functions of the monomer with functionality $>2$.

Operatively, a series of reactions with progressively larger excess of double bonds were carried out for 6 days, and the reaction with the minimum double bonds excess inhibiting gelation of the polymer led to the $r_{c}$ value for that couple of monomers. The $r_{c}$ values found for the reactions involving the couples $\mathrm{BP} / \mathrm{CYST}$ and CBA/EDA were 0.3083 and 0.3312 , respectively.

${ }^{1} \mathrm{H}$ NMR spectra confirmed the expected structures of linear and hyperbranched polymers. Moreover, there was no evidence of residual acrylamide double bonds, which usually result in cytotoxicity of the polymer. The ${ }^{1} \mathrm{H}$ NMR spectra of the polymers HB1-ETA, L1-ETA, HB2-ABOL and L2-ABOL, are given in Fig. S1a-d of the Supplementary Materials section 4.

All the synthesized PAAs showed good solubility in water and in buffer solutions. In the case of hyperbranched polymers, the formation of insoluble aggregates at the end of the reaction was noticed. These particles were filtered off by ultrafiltration with a 100,000 Da cut-off membrane. The average molecular weight was measured by GPC with online Refractive Index (RI) and UV detectors, and a Light Scattering (LALS) dual detector, using as mobile phase a $0.1 \mathrm{M}$ Tris buffer solution at $\mathrm{pH} 8.00 \pm 0.05$ with $0.2 \mathrm{M}$ sodium chloride. In the elution conditions, the absence of polymeric nanoparticles in the range 5-5000 nm was verified by DLS analysis. The number average molecular weight of the obtained PAAs ranged from 7800 to $16900 \mathrm{~g} / \mathrm{mol}$, (Table 1).

The GPC data related to the obtained PAAs are summarized in Table 1. From the GPC data it appears that there are no major differences in the absolute molecular weight between the linear and the branched PAAs.

It has been shown that high buffer capacity can promote endosomal escape of polymer/DNA polyplexes by the so-called "proton sponge effect" [35]. Therefore, the buffer capacity of the polymers, defined as the percentage of amine groups that become protonated during endosomal acidification in the $\mathrm{pH}$ range from $\mathrm{pH} 7.4$ (physiological pH) to 5.1 (late endosome), is a relevant parameter in the overall transfection process.

Table 1

GPC data and buffer capacity of the synthesized PAAs.

\begin{tabular}{|c|c|c|c|c|c|c|c|}
\hline & Polymer & $\bar{M}_{p}$ & $\bar{M}_{n}$ & $\bar{M}_{w}$ & PD & Yield & $\begin{array}{c}\text { Buffer } \\
\text { capacity }\end{array}$ \\
\hline & & $\begin{array}{c}(\mathrm{g} / \\
\mathrm{mol})\end{array}$ & $\begin{array}{c}(\mathrm{g} / \\
\mathrm{mol})\end{array}$ & $\begin{array}{c}(\mathrm{g} / \\
\mathrm{mol})\end{array}$ & & (\%) & (\%) \\
\hline \multirow[t]{4}{*}{ Hyperbranched } & HB1-ABOL & 9400 & 10,800 & 16,300 & 1.51 & 40 & 38 \\
\hline & HB1-ETA & 12,900 & 16,900 & 22,700 & 1.34 & 44 & 31 \\
\hline & HB2-ABOL & 6100 & 7800 & 14,100 & 1.80 & 38 & 18 \\
\hline & HB2-ETA & 8900 & 11,300 & 16,400 & 1.45 & 35 & 19 \\
\hline \multirow[t]{5}{*}{ Linear } & L1-ABOL & 9300 & 9600 & 14,800 & 1.54 & 87 & 23 \\
\hline & L1-ETA & 14,200 & 14,500 & 20,100 & 1.39 & 76 & 32 \\
\hline & L2-ABOL & 12,900 & 12,500 & 18,200 & 1.46 & 75 & 24 \\
\hline & L2-ETA & 10,700 & 10,500 & 14,800 & 1.41 & 78 & 32 \\
\hline & $\begin{array}{l}\text { Exgen500 } \\
\text { (linear PEI, } \\
22 \mathrm{kDa} \text { ) }\end{array}$ & - & - & - & - & - & 22 \\
\hline
\end{tabular}


The buffer capacity of the synthesized PAAs, together with Exgen 500 (linear PEI, $22 \mathrm{kDa}$ ) as a reference, is shown in Table 1 . The buffer capacity of the tested PAAs was higher compared to that of the reference polymer with the exception of HB2. A clear correlation between the terminal group and the buffer capacity value was not evident, since ETA-terminated polymers showed slightly higher values than ABOL terminated polymers in the case of linear PAAs but comparable or lower values for hyperbranched PAAs. In the case of hyperbranched polymers, CYST containing PAAs showed a higher buffer capacity than EDA containing PAAs which may be attributed to the lower $\mathrm{pK}_{\mathrm{a}}$ value of CYST compared to EDA in the polymeric chain [27] caused by the inductive effect of the disulfide group in the cystamine moiety, which shifts the $\mathrm{pK}_{\mathrm{a}}$ into the relevant buffer region. However, it is difficult to discern all the factors affecting the buffer capacity, the most critical being the structural and environmental differences of the internal and terminal amines and the tendency of the polymers to undergo $\mathrm{pH}$-dependent conformational changes and self-assembling in solution [29]. Therefore, no further general conclusions can be drawn on the effect of hyperbranching on the buffer capacity.

The degradability of the polymers in a reductive environment was also verified by ${ }^{1} \mathrm{H}$ NMR spectroscopy. In particular, the polymer solutions in $\mathrm{D}_{2} \mathrm{O}$ (0.1 $\mathrm{M}$ of disulfide linkages) were incubated with 2.4 equivalents of mercaptoethanol with respect to the concentration of disulfide linkages in the solution [37]. After $30 \mathrm{~min}$, both hyperbranched and linear PAAs were completely degraded. Thus, it can be deduced that the hyperbranching does not significantly affect the reductive degradation rate of PAAs.

\subsection{DNA complexation and release}

As free DNA is degraded in the extracellular environment and is poorly endocytosed, cationic polymers need to condense DNA to form polyplexes and protect and deliver DNA through the cell membrane. Moreover, it is known that positively charged polyplexes with size smaller than $200 \mathrm{~nm}$ are efficiently endocytosed [45]. To investigate whether these polymers were capable of DNA condensation, polymer-DNA polyplexes were prepared of polymer/DNA weight ratios $1 / 1,3 / 1,12 / 1$ and $48 / 1$, and the particle size and zeta-potential of these polyplexes were determined by DLS analysis. The results are shown in Fig. 1a and b.

In general, the hyperbranched polymers yielded smaller polyplexes than the linear polymers except at low $1 / 1$ and 3/1 polymer/DNA weight ratio. All the polyplexes displayed positive values of zeta potential (from $+10 \mathrm{mV}$ to $+22 \mathrm{mV}$ ) at polymer/DNA weight ratios equal or higher than $3 / 1$, whereas neutral or negative values at $1 / 1$ ratio indicate that DNA is not efficiently encapsulated at this ratio.

In the case of BP-containing PAAs, the hyperbranched polymers (HB1) showed a higher ability to shield the anionic charge of DNA, forming polyplexes with zeta potentials somewhat more positive than those of the linear counterparts (L1) at polymer/DNA ratios of 3/1 and higher. Remarkable is that L1-ABOL formed generally larger aggregates than L1-ETA, with lower values of $Z$ potential at all ratios. These differences in behavior could be due to effects of the terminal groups, although this is mirrored by the hyperbranched counterparts with a smaller difference between ABOL and ETA terminated polymers.

The CBA polymers (HB2, L2), formed smaller polyplexes than the BP polymers, particularly at low polymer/DNA weight ratios. This smaller particle size reflects the higher conformational flexibility of the CBA polymers which have much more rotational freedom in their bisacrylamide unit than the BP polymers. Moreover, the amino units in the CBA polymers (EDA and DMEDA) have a higher $\mathrm{pK}_{\mathrm{a}}$ than their disulfide-containing counterparts (CYST and DMC) in BP containing polymers [27], providing the CBA polymers a higher fraction of protonated amino groups, and thus exerting higher charge attraction with DNA, at the $\mathrm{pH} 7.4$ where polyplexes were prepared. A clear effect of hyperbranching on polyplex forming ability is however not apparent
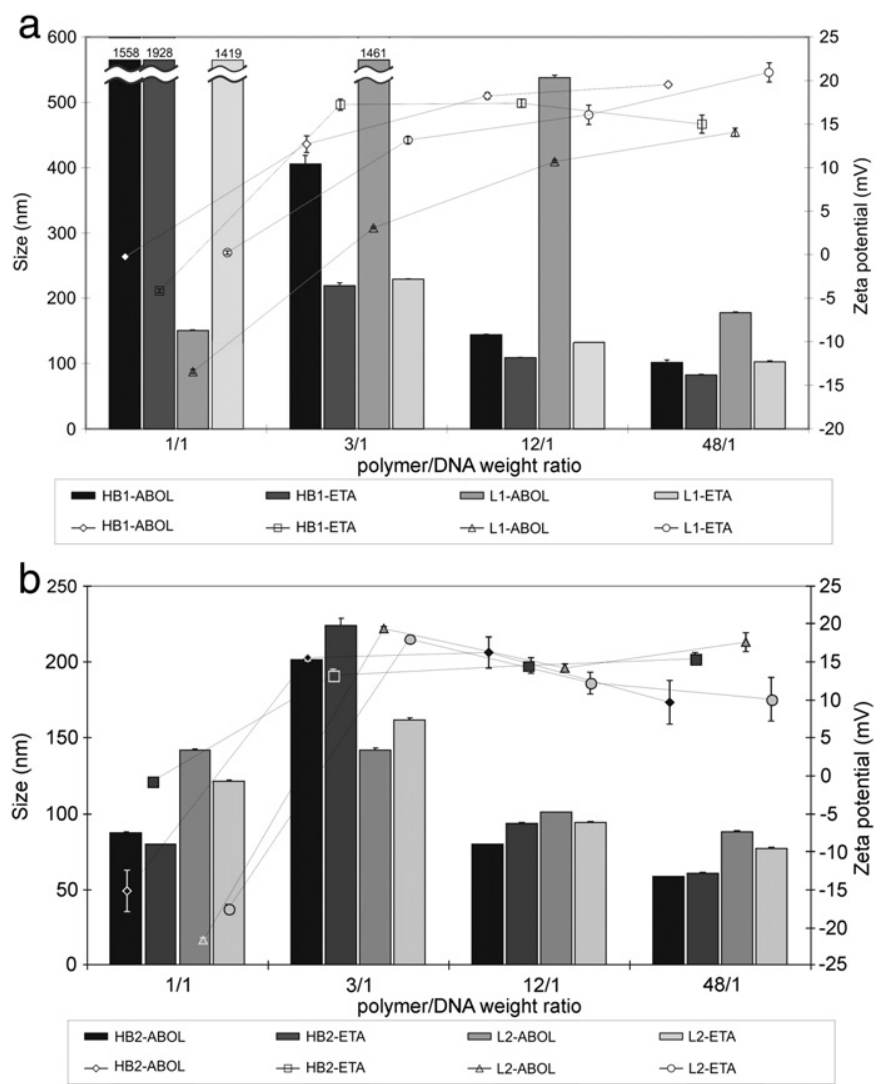

Fig. 1. (a) Particle size (depicted in bars) and corresponding zeta-potential values (depicted in symbols, lines are added for clarity) of the BP-containing PAAs. (b) Particle size (depicted in bars) and zeta-potential values (depicted in symbols) of the CBA-containing PAAs. Data are expressed as mean values of three determinations and the standard deviation is depicted in the error bars.

and these results show that many factors, like the nature of the terminal group and hydrophilic/hydrophobic balance, may result in evident differences in polymer/DNA polyplex formation.

Since negative values of zeta potential and particle sizes higher than $200 \mathrm{~nm}$ are usually related to poor transfection efficiency, the polyplexes formed at 1/1 polymer/DNA ratio were excluded from further investigation. To confirm the effective shielding of the DNA within the polyplexes against ethidium bromide intercalation, an agarose gel electrophoresis assay was performed on polymer-DNA polyplexes of the linear and hyperbranched polymers. As an example, the DNA retardation results by the hyperbranched and the linear CBA-containing polymers are depicted in Fig. 2.

In Fig. 2, lane 9 the free DNA is clearly visible as a band for super-coiled pDNA and a smaller band for the open circular form. The linear polymer L2-ABOL shows DNA retardation at all polymer/DNA weight ratios, whereas the hyperbranched polymer HB2-ABOL does the same, except at 3/1 polymer/DNA weight ratio. Comparing these data with the data in Fig. 1, where it can be observed that the hyperbranched polymer HB2-ABOL does form positively charged polyplexes from 3/1 polymer/DNA weight ratio, the data in Fig. 2 suggest that the linear polymer $\mathrm{L} 2-\mathrm{ABOL}$ is somewhat more efficient in DNA retardation than the related hyperbranched polymer at low polymer/DNA ratios. Despite the observation that with both polymers at $3 / 1$ polymer/DNA weight ratio polyplexes are formed, and the DNA is retarded during the electrophoresis, the DNA shielding against ethidium bromide intercalation is less efficient for the hyperbranched polymer, which may be attributed to the decreased polymer flexibility compared to the linear architecture [46]. A similar behavior was shown by HB2-ETA and L2-ETA polymers, whereas, in contrast, HB1 and L1 based polymers showed efficient DNA retardation at all 


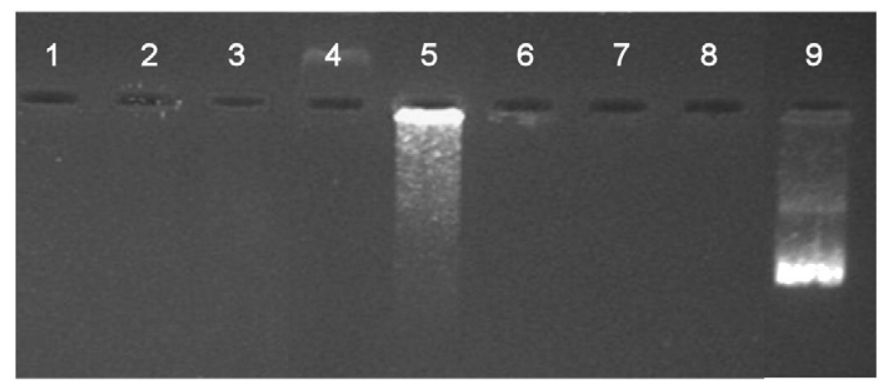

Fig. 2. Agarose gel retardation of plasmid DNA by hyperbranched and linear CBA based polymer. From left to right are shown polyplexes of linear polymer L2-ABOL at 3/1 (lane 1), 6/1 (lane 2), 12/1 (lane 3) and 48/1 (lane 4) polymer/DNA weight ratio. Next, polyplexes of the hyperbranched polymer HB2-ABOL at 3/1 (lane 5), 6/1 (lane 6), 12/1 (lane 7) and 48/1 (lane 8) polymer/DNA weight ratio. pDNA as control is shown in lane 9 .

polymer/DNA weight ratio (data not shown). Based on these results, we performed the DNA transfection tests on polyplexes with polymer/DNA ratio from $3 / 1$ to $48 / 1$.

\subsection{In vitro cell viability and transfection efficiency}

Cytotoxicity is an important parameter to be evaluated in the development of safe non-viral vectors and is determined by many factors like molecular weight, charge density, type of amines, polymer structure (linear, branched), intracellular degradability and chain flexibility. High cytotoxicity caused by the vector will negatively affect the transfection process. To investigate the cytotoxicity of the polyplexes, the cell viability of COS-7 cells (Green African monkey kidney) was determined by MTT assay in transfection experiments with polyplexes with pCMV-LacZ plasmid prepared at 3/1,6/1,12/1 and 48/1 polymer/DNA weight ratios. Cell viability tests were performed both in presence and absence of $10 \%(\mathrm{v} / \mathrm{v})$ fetal bovine serum (FBS), and the results are shown in Fig. 3.
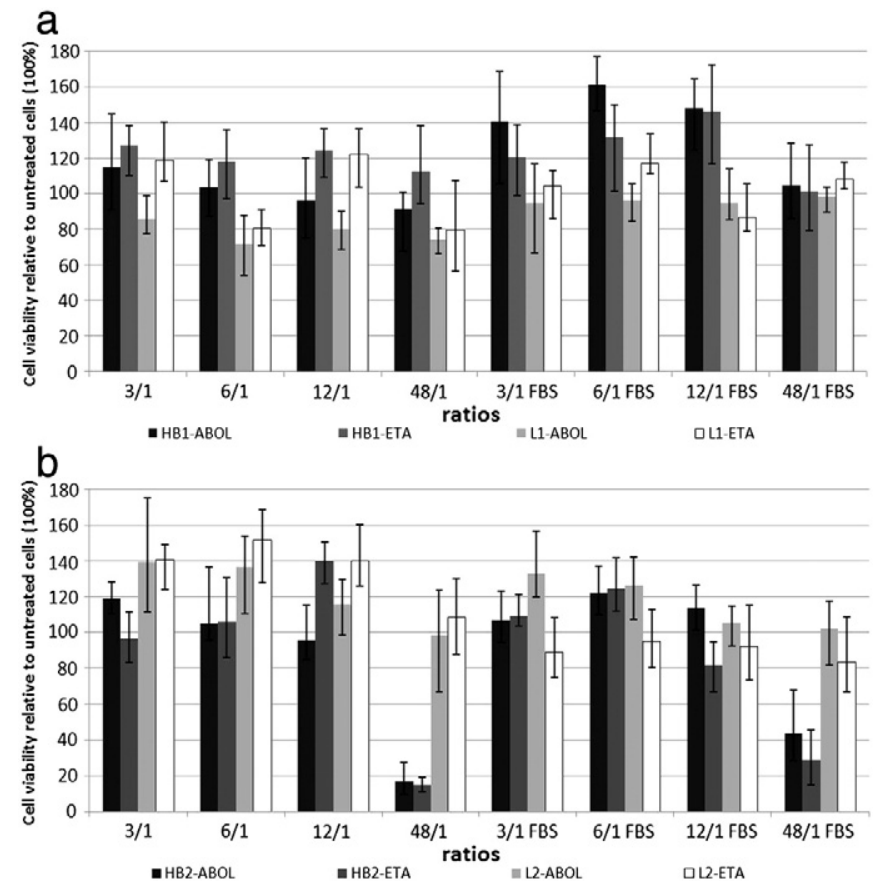

Fig. 3. Cell viabilities of polyplexes of BP-containing polymers (a) and CBA-containing polymers (b) at 3/1,6/1, 12/1 and 48/1 polymer/DNA weight ratios, in absence and presence of fetal bovine serum (FBS, right part of the figures).
All the BP polymers give polyplexes with only low or no cytotoxicity; the hyperbranched HB1 polyplexes generally show somewhat less cytotoxicity than the related linear L1 polyplexes. The presence of serum resulted in some enhancement of cell viability, which is most apparent for the hyperbranched polyplexes. Also the CBA-containing polyplexes displayed only low or no cytotoxicity, both under serum-free and serum conditions, except for the hyperbranched HB2 polyplexes at the highest $48 / 1$ polymer/DNA weight ratio, for which significant cytotoxicity is observed. This cytotoxic behavior could be due to the relatively high degree of protonation, and thus relatively high cationic charge, of the HB2 polymers, causing interference with intracellular membrane processes. Also the presence of a relatively large fraction of free HB2 polymer can contribute to the cytotoxicity observed in this case. In the series of tested SS-PAA polymers, no general trend on the cell viability caused by the terminal groups (ABOL and ETA) is apparent.

In order to evaluate whether hyperbranching has a beneficial effect on the overall transfection efficiency, the transfection of polyplexes based on the synthesized PAAs was studied in vitro by using COS-7 cells. Transfection efficiencies were measured by ONPG assay under the same conditions as used in the cell viability tests. Polyplexes based on Exgen 500 (linear PEI) at its optimal N/P ratio of 5/1 were used as reference. Transfection efficiencies for all the polymers tested are presented in Fig. 4.

Comparison of the data of the linear and hyperbranched PAAs (Fig. 4a,b) shows that the hyperbranched PAAs give generally higher transfection efficiencies. Moreover it can be noticed that the transfection efficiency in the presence of serum (10\% v/v FBS) was generally higher than in the absence of serum. The enhanced transfection efficiency for the hyperbranched PAAs could at least partially be due to the somewhat higher cell viability displayed with these polymers in the presence of FBS (Fig. 3).
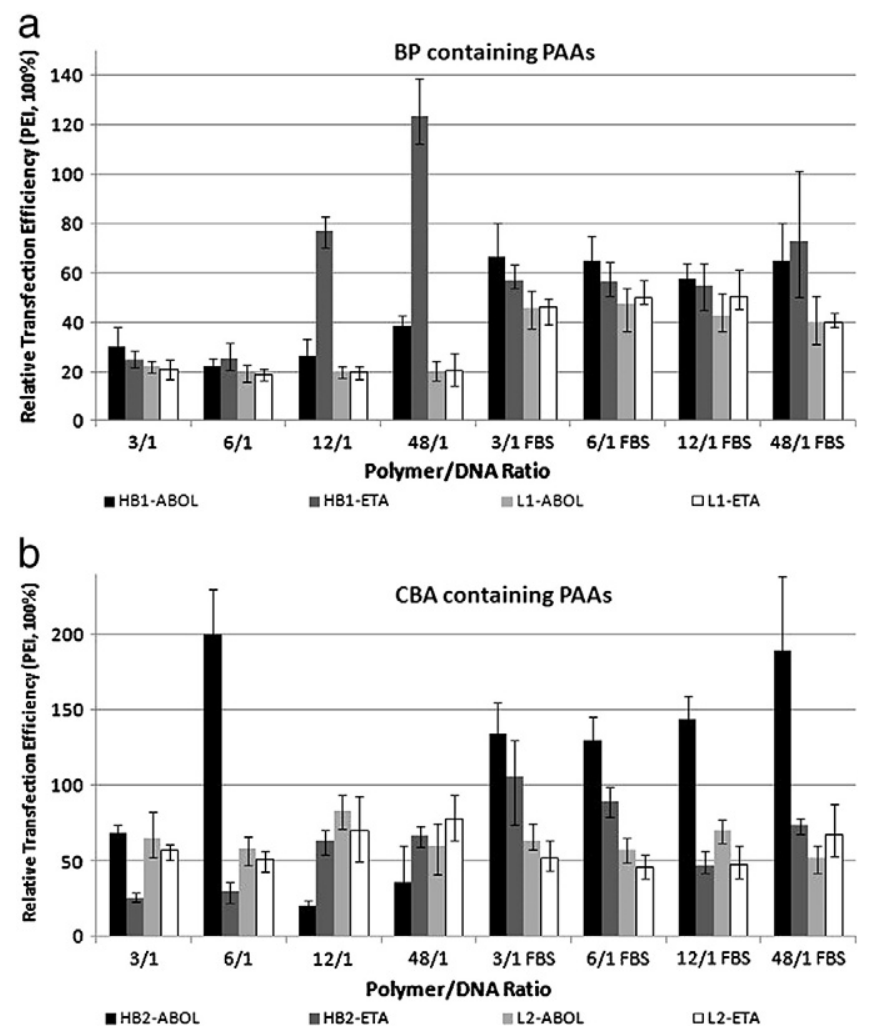

Fig. 4. DNA transfection efficiency of polyplexes hyperbranched polymers (black and dark gray bars) versus linear polymers (gray and white bars) at polymer/DNA ratio $3 / 1,6 / 1,12 / 1$ and $48 / 1$ in absence and in presence of FBS: (a) BP containing PAAs; (b) CBA-containing PAAs. 
Among the BP-containing PAAs, the linear polymers only showed low transfection efficiency both in the absence and in the presence of serum. Among the hyperbranched BP polymers, only the polyplexes of HB1-ETA at polymer/DNA ratios of $12 / 1$ and $48 / 1$ in absence of FBS showed appreciable transfection efficiency, comparable with PEI. Among the CBA-containing PAAs, HB2-ABOL showed the highest transfection efficiency both without serum (at 6/1 polymer/DNA weight ratio) and with serum (at all ratios). In the medium without serum, the decrease in transfection efficiency of HB2-ABOL at 12/1 and 48/1 polymer/DNA ratio is probably due to the increased cytotoxicity in the presence of excess of this polymer. In the presence of serum, the toxicity of polyplexes probably becomes reduced by protein complexation and the transfection efficiency outperformed the PEI efficiency at all ratios.

From the data in Fig. $4 a$ and $b$ it can be seen that the transfection of polyplexes of CBA-containing PAAs is more effective than those of BP-containing PAAs, which is in accordance with the results of our previous work [27]. It may be speculated that the higher flexibility of the CBA polymers allows for increased membrane disruptive interactions upon endosomal acidification, leading to better endosomal escape of the polyplexes.

With respect to the terminal function, for CBA-containing polymers ABOL showed in general the highest efficiencies (Fig. 4), whereas for BP-containing polymers only in two cases (HB1-ETA at 12/1 and 48/1 polymer/DNA weight ratio) ETA exhibited higher transfection efficiency than ABOL. Moreover, it was observed that the difference in transfection efficiency between ABOL and ETA terminated polymers is enhanced in the case of hyperbranched PAAs. This was expected from the higher number of terminal functions per repeating unit of the hyperbranched polymers as compared to their linear analogs. However, in the case of BP-containing PAAs this effect was not generally observed, probably because of the low transfection efficiency of most of the polymers tested. These results are in accordance with the findings of Zugates and coworkers, in which the terminal groups were found to have an unexpectedly high influence on biophysical properties and transfection efficiency of linear poly(amino ester)s [36].

\subsection{Conclusions}

Four novel hyperbranched and four analogous linear PAAs containing disulfide linkages in the main chain were synthesized with different terminal functional groups (ABOL versus ETA). These polymers showed good properties for self-assembling polyplex formation, thereby condensing plasmid DNA in polyplexes smaller than $200 \mathrm{~nm}$ at 3/1 and higher polymer/DNA weight ratios.

The polyplexes from these disulfide containing PAAs have generally low or no toxicity and are capable to rapidly release of DNA by disulfide degradation in the reductive environment, as is present inside the cytoplasm. The transfection efficiencies of the hyperbranched polymers were examined and compared with their linear analogs. The CBA-containing polymers showed DNA transfection efficiencies higher than or comparable with PEI and performed mostly better than BPcontaining polymers. In general, polyplexes from hyperbranched PAAs yielded higher transfection efficiencies than those from their linear analogs. The highest transfection efficiency was obtained with the hyperbranched, ABOL terminated, CBA-containing PAA (HB2-ABOL). However, in this library of polymers direct correlations between hyperbranching, and transfection efficiency were not readily apparent. Most importantly, transfection efficiencies of the branched PAAs were generally enhanced by the presence of serum, which is a promising property for future in vivo studies with these hyperbranched PAAs.

In this study, the easy synthetic modifications of both linear and hyperbranched poly(amido amide)s and the versatility of hyperbranched PAAs in regulating DNA transfection and cytotoxicity is demonstrated. These hyperbranched polymers could provide new strategies for the use of poly(amido amine)s as smart polymeric vectors with tailorable properties in gene therapy.

\section{Appendix A. Supplementary data}

Supplementary data to this article can be found online at http:// dx.doi.org/10.1016/j.jconrel.2012.07.029.

\section{References}

[1] J.J. Green, R. Langer, D.G. Anderson, A combinatorial polymer library approach yields insight into nonviral gene delivery, Acc. Chem. Res. 41 (2008) 749-759.

[2] S. McTaggart, M. Al-Rubeai, Retroviral vectors for human gene delivery, Biotechnol. Adv. 20 (2002) 1-31.

[3] D.C. Gorecki, Prospects and problems of gene therapy: an update, Expert Opin. Emerg. Drugs 6 (2001) 187-198.

[4] S. Lehrman, Virus treatment questioned after gene therapy death, Nature 401 (1999) 517-518.

[5] Q. Liu, D.A. Muruve, Molecular basis of the inflammatory response to adenovirus vectors, Gene Ther. 10 (2003) 935-940.

[6] J.Y. Sun, V. Anand-Jawa, S. Chatterjee, K.K. Wong, Immune responses to adeno-associated virus and its recombinant vectors, Gene Ther. 10 (2003) 964-976.

[7] R.E. Donahue, S.W. Kessler, D. Bodine, K. McDonagh, C. Dunbar, S. Goodman, B. Agricola, E. Byrne, M. Raffeld, R. Moen, Helper virus induced T cell lymphoma in nonhuman primates after retroviral mediated gene transfer, J. Exp. Med. 176 (1992) 1125-1135.

[8] M.E. Gore, Adverse effects of gene therapy: gene therapy can cause leukaemia: no shock, mild horror but a probe, Gene Ther. 10 (2003) 4

[9] X. Zhang, W.T. Godbey, Viral vectors for gene delivery in tissue engineering, Adv. Drug Deliv. Rev. 58 (2006) 515-534.

[10] J. Luten, C.F. van Nostrum, S.C. De Smedt, W.E. Hennink, Biodegradable polymers as non-viral carriers for plasmid DNA delivery, J. Control. Release 126 (2008) 97-110.

[11] P. Dubruel, E. Schacht, Vinyl polymers as non-viral gene delivery carriers: current status and prospects, Macromol. Biosci. 6 (2006) 789-810

[12] M.D. Brown, A.G. Schatzlein, I.F. Uchegbu, Gene delivery with synthetic (non-viral) carriers, Int. J. Pharm. 229 (2001) 1-21.

13] T. Merdan, J. Kopecek, T. Kissel, Prospects for cationic polymers in gene and oligonucleotide therapy against cancer, Adv. Drug Deliv. Rev. 54 (2002) 715-758.

[14] S.C. De Smedt, J. Demeester, W.E. Hennink, Cationic polymer based gene delivery systems, Pharm. Res. 17 (2000) 113-126.

[15] G.A. Pietersz, C.K. Tang, V. Apostolopoulos, Structure and design of polycationic carriers for gene delivery, Mini Rev. Med. Chem. 6 (2006) 1285-1298.

[16] C. Louise, Nonviral vectors, Methods Mol. Biol. 333 (2006) 201-226.

[17] J.K. Vasir, V. Labhasetwar, Polymeric nanoparticles for gene delivery, Expert Opin. Drug Deliv. 3 (2006) 325-344.

[18] P. Ferruti, M.A. Marchisio, R. Duncan, Poly(amido-amine)s: biomedical applications, Macromol. Rapid Commun. 23 (2002) 332-355.

[19] S. Richardson, P. Ferruti, R. Duncan, Poly(amido amine)s as potential endosomolytic polymers: evaluation in vitro and body distribution in normal and tumour-bearing animals, J. Drug Target. 6 (1999) 391-404

[20] S.C.W. Richardson, N.G. Pattrick, Y.K.S. Man, P. Ferruti, R. Duncan, Poly(amido amine) s as potential nonviral vectors: ability to form interpolyelectrolyte complexes and to mediate transfection in vitro, Biomacromolecules 2 (2001) 1023-1028.

[21] For reviews see: (a) C. Lin, J.F.J. Engbersen, The role of the disulfide group in disulfide-based polymeric gene carriers, Expert Opin. Drug Deliv. 6 (2009), 421-439; (b) F.H. Meng. W.E. Hennink, Z.Y. Zhong, Reduction-sensitive polymers and bioconjugates for biomedical applications, Biomaterials 30 (2009) 2180-2198.

[22] R. Cheng, F.H. Meng, C. Deng, J. Feijen, Z.Y. Zhong, Glutathione-responsive nano-vehicles as a promising platform for targeted intracellular drug and gene delivery, J. Control. Release 152 (2011) 2-12.

[23] Y.H. Wang, M. Zheng, F.H. Meng, J. Zhang, R. Peng, Z.Y. Zhong, Branched polyethylenimine derivatives with reductively cleavable periphery for safe and efficient in vitro gene transfer, Biomacromolecules 12 (2011) 1032-1040.

[24] E. Emilitri, P. Ferruti, R. Annunziata, E. Ranucci, Novel amphoteric cystine-based poly(amido amine)s responsive to redox stimuli, Macromolecules 40 (2007) 4785-4793.

[25] C. Lin, Z.Y. Zhong, M.C. Lok, X.L. Jiang, W.E. Hennink, J. Feijen, J.F.J. Engbersen, Linear poly(amido amine)s with secondary and tertiary amino groups and variable amounts of disulfide linkages: synthesis and in vitro gene transfer properties, J. Control. Release 116 (2006) 130-137.

[26] C. Lin, Z. Zhong, M.C. Lok, X. Jiang, W.E. Hennink, J. Feijen, J.F.J. Engbersen, Novel bioreducible poly(amido amine)s for highly efficient gene delivery, Bioconjug. Chem. 18 (2007) 138-145.

[27] M. Piest, C. Lin, M.A. Mateos-Timoneda, M.C. Lok, W.E. Hennink, J. Feijen, J.F.J Engbersen, Novel poly(amido amine)s with bioreducible disulfide linkages in their diamino-units: structure effects and in vitro gene transfer properties, J. Control. Release 130 (2008) 38-45.

[28] C. Lin, C.J. Blaauboer, M.M. Timoneda, M.C. Lok, M. van Steenbergen, W.E. Hennink, Z.Y. Zhong, J. Feijen, J.F.J. Engbersen, Bioreducible poly(amido amine)s with oligoamine side chains: synthesis, characterization, and structural effects on gene delivery, J. Control. Release 126 (2008) 166-174.

[29] M.A. Mateos-Timoneda, M.C. Lok, W.E. Hennink, J. Feijen, J.F.J. Engbersen, Poly(amido amine)s as gene delivery vectors: effects of quaternary nicotinamide moieties in the side chains, ChemMedChem 3 (2008) 478-486. 
[30] M. Piest, J.F.J. Engbersen, Effects of charge density and hydrophobicity of poly(amido amine)s for non-viral gene delivery, J. Control. Release 148 (2010) 83-90.

[31] J. Chen, C. Wu, D. Oupicky, Bioreducible poly(amido amines)s for gene delivery, Biomacromolecules 10 (2009) 2921-2927.

[32] J. Dennig, Gene transfer in eukaryotic cells using activated dendrimers, Top. Curr. Chem. 228 (2003) 227-236.

[33] Z. Zhong, Y. Song, J.F.J. Engbersen, M.C. Lok, W.E. Hennink, J. Feijen, A versatile family of degradable non-viral gene carriers based on hyperbranched poly(ester amine)s, J. Control. Release 109 (2005) 317-329.

[34] R.B. Wang, L.Z. Zhou, Y.F. Zhou, G.L. Li, X.Y. Zhu, H.C. Gu, X.L. Jiang, H.Q. Li, J.L. Wu, L. He, X.Q. Guo, B.S. Zhu, D.Y. Yan, Synthesis and gene delivery of poly(amido amine)s with different branched architecture, Biomacromolecules 11 (2010) 489-495.

[35] J.P. Behr, The proton sponge: a trick to enter cells the viruses did not exploit, Chimia 51 (1997) 34-36.

[36] G.T. Zugates, N.C. Tedford, A. Zumbuehl, S. Jhunjhunwala, C.S. Kang, L.G. Griffith, D.A. Lauffenburger, R. Langer, D.G. Anderson, Gene delivery properties of end-modified polyo(beta-amino ester)s, Bioconjug. Chem. 18 (2007) 1887-1896.

[37] E. Emilitri, E. Ranucci, P. Ferruti, New poly(amidoamine)s containing disulfide linkages in their main chain, J. Polym. Sci. A Polym. Chem. 43 (2005) 1404-1416.

[38] J.Y. Cherng P. vande Wetering H. Talsma, D.J.A. Crommelin, W.E. Hennink, Effect of size and serum proteins on transfection efficiency of poly((2-dimethylamino) ethyl methacrylate)-plasmid nanoparticles, Pharm. Res. 13 (1996) 1038-1042.
[39] C. Arigita, N.J.Zuidam, D.J.A. Crommelin, W.E. Hennink, Association and dissociation characteristics of polymer/DNA complexes used for gene delivery, Pharm. Res. 16 (1999) 1534-1541.

[40] D.A. Scudiero, R.H. Shoemaker, K.D. Paull, A. Monks, S. Tierney, T.H. Nofziger, M.] Currens, D. Seniff, M.R. Boyd, Evaluation of a soluble tetrazolium formazan assay for cell-growth and drug sensitivity in culture using human and other tumor-cell lines, Cancer Res. 48 (1988) 4827-4833.

[41] P. Ferruti, E Ranucci, F. Trotta, E Gianasi, E.G. Evagorou, M. Wasil, G Wilson, R. Duncan, Synthesis, characterisation and antitumour activity of platinum(II) complexes of novel functionalised poly(amido amine)s, Macromol. Chem. Phys. 200 (1999) 1644-1654.

[42] G. Odian, in: Principles of Polymerization, Wiley, New York, 1991, pp. 40-185.

[43] P.J. Flory, Principles of Polymer Chemistry, Cornell University Press, 1953.

[44] P. Ferruti, E. Ranucci, F. Trotta, R. Cavalli, Polimeri iperramificati a base di ciclodestrine e poli(amidoammine) per il rilascio controllato di farmaci insolubili, Italian Patent $n^{\circ}$ MI2007A1173, 2007.

[45] S. Grosse, Y. Aron, G. Thevenot, D. Francois, M. Monsigny, I. Fajac, Potocytosis and cellular exit of complexes as cellular pathways for gene delivery by polycations, J. Gene Med. 7 (2005) 1275-1286.

[46] L. Wightman, R. Kircheis, V. Rossler, S. Carotta, R. Ruzicka, M. Kursa, E. Wagner Different behavior of branched and linear polyethylenimine for gene delivery in vitro and in vivo, J. Gene Med. 3 (2001) 362-372. 